\title{
Comparison of Adverse Event Profiles of Tumor Necrosis Factor-Alfa Inhibitors: Analysis of a Spontaneous Reporting Database
}

This article was published in the following Dove Press journal: Therapeutics and Clinical Risk Management

\section{Tomohito Wakabayashi \\ Keiko Hosohata (ID \\ Saki Oyama \\ Ayaka Inada \\ Sayaka Ueno \\ Hiroko Kambara \\ Tatsuya lida \\ Takahiro Nakatsuji \\ Mayako Uchida (iD) \\ Kazunori Iwanaga}

Education and Research Center for Clinical Pharmacy, Osaka University of Pharmaceutical Sciences, Takatsuki, Osaka, Japan
Correspondence: Keiko Hosohata Education and Research Center for Clinical Pharmacy, Osaka University of Pharmaceutical Sciences, 4-20-I

Nasahara, Takatsuki, Osaka 569-1094, Japan

Tel +8I-72-690-|27|

Fax +81-72-690-1023

Email hosohata@gly.oups.ac.jp
Introduction: Concerns over safety profiles of tumor necrosis factor (TNF)-alfa inhibitors have been raised. The purpose of this study was to clarify the adverse events associated with TNF-alfa inhibitors using a spontaneous reporting system database.

Materials and Methods: A retrospective pharmacovigilance disproportionality analysis was conducted using the Japanese Adverse Drug Event Report (JADER) database. Adverse event reports submitted to the Pharmaceuticals and Medical Devices Agency between 2004 and 2017 were analyzed, and the reporting odds ratio (ROR) and 95\% confidence interval (CI) for each adverse event were calculated.

Results: Among the 34,031 reports of adverse events associated with TNF-alfa inhibitors, $65.8 \%$ were women, who were frequently in their $60 \mathrm{~s}(28.2 \%)$. Signals were detected for pneumonia (ROR, 5.36; 95\% CI, 5.14-5.6), interstitial lung disease (ROR, 2.04; 95\% CI, 1.95-2.15), pneumocystis jirovecii pneumonia (ROR, 11.8; 95\% CI, 11.1-12.5), and herpes zoster (ROR, 6.4; 95\% CI, 5.92-6.91) for TNF-alfa inhibitors as a class. There was variability in their signal strength across individual TNF-alfa inhibitors.

Conclusion: The strength of the associations of TNF-alfa inhibitors with adverse events is variable, and further studies are required to evaluate the identified signals.

Keywords: TNF-alfa inhibitors, adverse drug events, spontaneous reporting system, reporting odds ratio, Japanese Adverse Drug Event Report database

\section{Introduction}

Tumor necrosis factor (TNF)-alpha is a potent pro-inflammatory cytokine exerting pleiotropic effects on various cell types and plays a central role in the pathogenesis of inflammatory diseases. Antibodies that bind to and neutralize TNF-alfa have been developed in order to inhibit its activity, and have been shown to be effective for patients with rheumatoid arthritis (RA) and other forms of inflammatory disease such as psoriasis, psoriatic arthritis, juvenile rheumatoid arthritis (JRA), ankylosing spondylitis (AS), and inflammatory bowel disease (IBD). ${ }^{1,2}$ Currently available therapies for them are infliximab, etanercept, adalimumab, golimumab, and certolizumab pegol in Japan. Several Phase III studies showed that TNF-alpha inhibitors had favorable safety profiles. ${ }^{3,4}$ In clinical practice, however, unexpected adverse events could occur because patients have various backgrounds and etiologies, unlike in clinical trials, where enrollment criteria are strict. Therefore, unexpected adverse drug effects can emerge, and so investigation of their occurrence is important. 
In the post-marketing phase, it is important to monitor high-priority adverse events and gain insight into actual drug safety profiles. Spontaneous reporting systems are a primary source of information to detect safety signals, especially for newly marketed drugs. ${ }^{5,6}$ For the pharmacovigilance approach, the Japanese Adverse Drug Event Report (JADER) database is a large published database managed by the Pharmaceuticals and Medical Devices Agency (PMDA). ${ }^{7,8}$ In this study, we aimed to clarify the adverse event profiles of five TNF-alfa inhibitors as a class and individual agents in real-world settings using the JADER database.

\section{Methods}

The JADER database is freely obtainable from the website of the PMDA, which has been reported. ${ }^{9-12}$ The data covered the period between April 2004 and January 2017. The JADER consists of 4 tables: patient demographic information (DEMO), drug information (DRUG), adverse events (REAC), and medical history. After we removed duplicate data from each table, the DEMO table was then linked to the REAC and DRUG tables using the ID number. The contribution of the medication to adverse events was classified into three categories: "suspected medicine," "concomitant medicine," and "interaction." We only extracted cases that were classified as "suspected medicine" and analyzed the reports of suspected drugs and adverse events in the "Preferred Term (PT)" coded in the Medical Dictionary for Regulatory Activities (MedDRA). We compiled a cross-tabulation table based on two classifications: the presence or absence of the adverse event, and the presence or absence of the suspected medicine. Then, we calculated the reporting odds ratio (ROR) by the following formula.

$$
\begin{aligned}
\mathrm{ROR} & =\frac{a / b}{c / d}, 95 \% \mathrm{CI} \\
& =\exp \left\{\log (\mathrm{ROR}) \pm 1.96 \sqrt{\frac{1}{a}+\frac{1}{b}+\frac{1}{c}+\frac{1}{d}}\right\}
\end{aligned}
$$

a: the number of patients with a target event when they received a target drug

$\mathrm{b}$ : the number of patients with non-target adverse events when they received a target drug

c: the number of patients with a target event when they received non-target drugs

$\mathrm{d}$ : the number of patients with non-target adverse events when they received non-target drugs

A signal was considered present when the lower limit of the $95 \%$ CI of the ROR exceeded one.

\section{Results}

The total number of adverse events associated with the use of TNF-alfa inhibitors was 34,031. Of those, 16,724, 7441, 5131,3376 , and 1359 were reported with infliximab, etanercept, adalimumab, golimumab, and certolizumab pegol, respectively (Table 1). Infliximab has been available for the longest period among the five drugs (Table S1). As shown in Table 2, characteristics of those who experienced adverse events on receiving TNF-alfa inhibitors are listed. In brief, most of the reports concerned females $(65.8 \%)$, who were most frequently in their $60 \mathrm{~s}(28.2 \%)$. Reasons for using TNF-alfa inhibitors included RA (71.3\%), Crohn's disease (13.0\%), and ulcerative colitis (3.0\%). Drug use for unknown or other indications involved 1620 reports (4.7\%).

Table 3 shows the disproportionality analysis based on the top 30 adverse events associated with TNF-alfa inhibitors as a class. The most frequently reported adverse event associated with TNF-alfa inhibitors as a class was pneumonia (ROR, 5.36; 95\% CI, 5.14-5.6), followed by

\begin{tabular}{|c|c|c|c|c|c|c|c|c|c|c|c|c|c|c|}
\hline & \multicolumn{13}{|l|}{ Year } & \multirow[t]{2}{*}{ Total } \\
\hline & 2004 & 2005 & 2006 & 2007 & 2008 & 2009 & 2010 & 2011 & 2012 & 2013 & 2014 & 2015 & 2016 & \\
\hline All TNF-alfa inhibitors & 2397 & 2744 & 2597 & 2620 & 1899 & 2196 & 2937 & 2380 & 3361 & 3576 & 2517 & 2427 & 2380 & 34,031 \\
\hline Infliximab & 2397 & 2041 & 1803 & 2025 & 1267 & 1115 & 1646 & 995 & 982 & 778 & 643 & 498 & 534 & 16,724 \\
\hline Etanercept & & 703 & 794 & 595 & 507 & 615 & 676 & 548 & 774 & 488 & 464 & 593 & 684 & $744 I$ \\
\hline Adalimumab & & & & & 125 & 466 & 615 & 794 & 570 & 443 & 798 & 726 & 594 & 5131 \\
\hline Golimumab & & & & & & & & 43 & 1009 & 1643 & 280 & 204 & 197 & 3376 \\
\hline Certolizumab pegol & & & & & & & & & 26 & 224 & 332 & 406 & 371 & 1359 \\
\hline
\end{tabular}

Table I Annual Reports of Adverse Events Associated with TNF-Alfa Inhibitors from 2004 to 2016

Abbreviation: TNF, tumor necrosis factor. 
Table 2 Characteristics of the Patients with Adverse Events Associated with TNF-Alfa Inhibitors

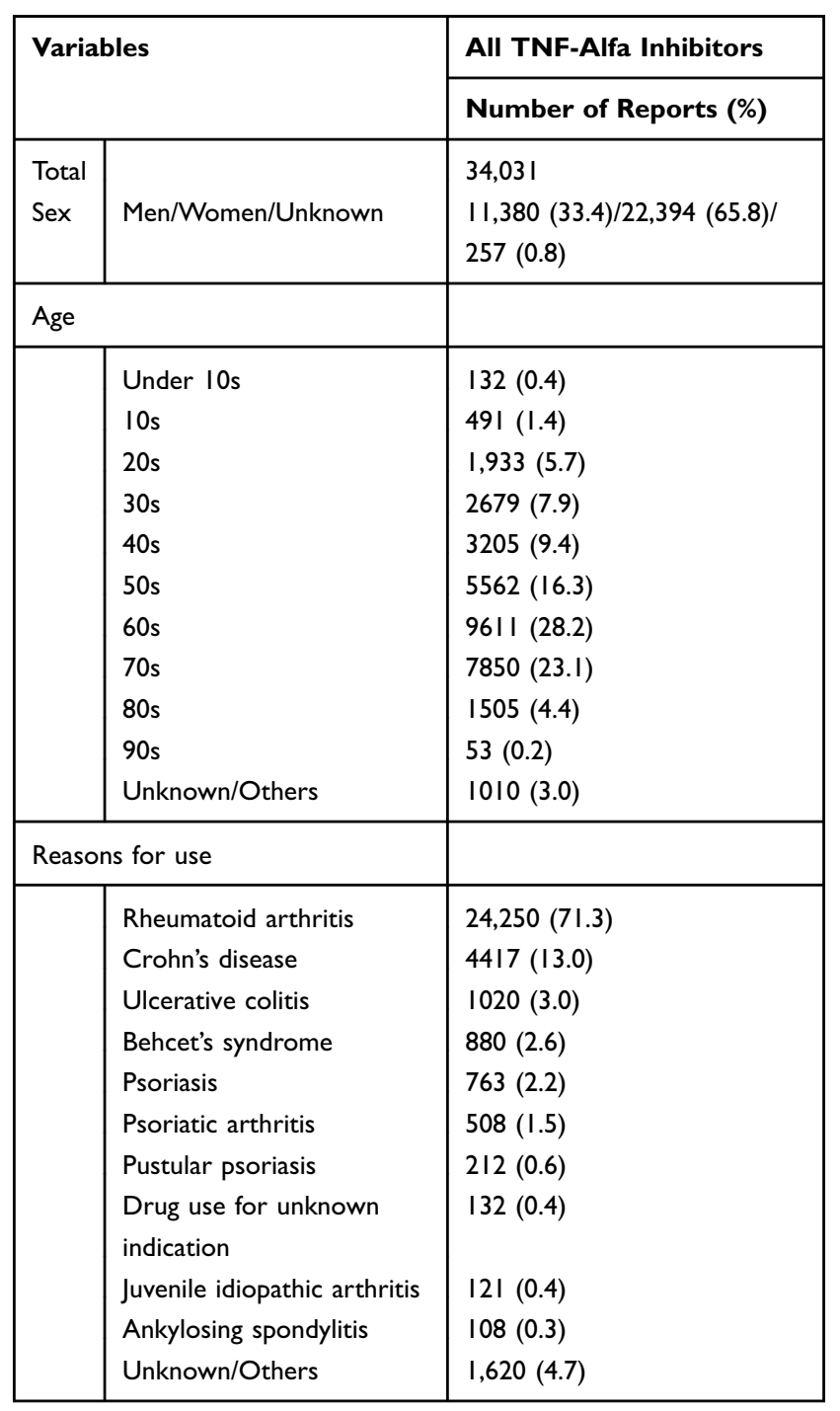

Abbreviation: TNF, tumor necrosis factor.

interstitial lung disease (ROR, 2.04; 95\% CI, 1.95-2.15), pneumocystis jirovecii pneumonia (ROR, $11.8 ; 95 \% \mathrm{CI}$, 11.1-12.5), herpes zoster (ROR, 6.4; 95\% CI, 5.92-6.91), infusion-related reaction (ROR, 29.2; 95\% CI, 26.6-32), sepsis (ROR, 3.16; 95\% CI, 2.92-3.41), and pneumonia bacterial (ROR, 11.5; 95\% CI, 10.5-12.5). Focusing on SOC, "infections and infestations" were most frequently reported.

Next, we examined the top 10 adverse events by individual TNF-alfa inhibitor (Tables 4-8). Pneumonia was the most frequently reported for infliximab, etanercept, adalimumab, golimumab, and certolizumab pegol. Especially, the number of reports involving infliximab was the highest (1254 reports). Interstitial lung disease ranked high in infliximab, etanercept, adalimumab, golimumab, and certolizumab, and the ROR values were similar among the five TNF-alfa inhibitors. The ROR value for pneumocystis jirovecii pneumonia suggested the strongest association with infliximab. As for infusion reactions, reports were obtained for only infliximab, and the ROR value was noteworthy (ROR, 58.6; 95\% CI, 53.4-64.3). Regarding malignant tumors, the associations of breast and colon cancers were suggested with etanercept and certolizumab pegol, respectively.

\section{Discussion}

This study presents profiles of adverse events associated with the use of TNF-alfa inhibitors based on the real-world data from the JADER database. In our results, the number of reports of adverse events involving TNF-alfa inhibitors during the study period were 34,031 , and there is variability in the safety profile among TNF-alfa inhibitors. To the best of our knowledge, this is the first study to report the associations of five TNF-alfa inhibitors with adverse events using a spontaneous reporting database.

Among the TNF-alfa inhibitors analyzed in this study, infliximab has been clinically available for use for the longest (Table S1), and so it is suggested that infliximab is likely to be associated with more adverse events, solely based on this longer time on the market. In this study, we found that reports of infectious adverse events were common on the use of TNF-alfa inhibitors (Table 3). TNF-alfa inhibitors suppress immunity; therefore, the risk of infection may be increased. ${ }^{13,14}$ However, we found that individual agents showed a variable signal strength for multiple types of infectious adverse events. For example, the ROR value for pneumocystis jirovecii pneumonia was the highest for infliximab, whereas that for herpes zoster was the highest for certolizumab pegol. On the other hand, interstitial lung disease, which was the second most highly reported after pneumonia (Table 3), showed almost the same signal values among all TNF-alfa inhibitors. Wakao et al reported that interstitial lung disease is more often reported in Japan compared with the rest of the world. ${ }^{15}$ There may be a coding preference that contributes to the difference in the relative reporting rate of interstitial lung disease between Japan and the rest of the world. Therefore, interstitial lung disease may be more commonly reported on the use of individual TNF-alfa inhibitors, leading to small variations in signal values.

Binding and neutralizing activities against soluble TNF are common actions of TNF-alfa inhibitors; however, recent studies demonstrated that these inhibitors 
Table 3 The Top 30 Adverse Drug Events Associated with TNF-Alfa Inhibitors

\begin{tabular}{|c|c|c|c|c|}
\hline \multirow[t]{2}{*}{ PT } & \multirow[t]{2}{*}{ soc } & \multicolumn{3}{|c|}{$\begin{array}{l}\text { All } \\
\text { TNF-Alfa Inhibitors }\end{array}$} \\
\hline & & $\mathbf{n}$ & ROR & $95 \% \mathrm{Cl}$ \\
\hline Pneumonia & Infections and infestations & 2517 & 5.36 & $5.14-5.6 *$ \\
\hline Interstitial lung disease & Respiratory, thoracic and mediastinal disorders & 1760 & 2.04 & $1.95-2.15^{*}$ \\
\hline Pneumocystis jirovecii pneumonia & Infections and infestations & 1357 & 11.8 & $11.1-12.5^{*}$ \\
\hline Herpes zoster & Infections and infestations & 729 & 6.4 & $5.92-6.91 *$ \\
\hline Infusion-related reaction & Injury, poisoning and procedural complications & 679 & 29.2 & $26.6-32 *$ \\
\hline Sepsis & Infections and infestations & 660 & 3.16 & $2.92-3.41 *$ \\
\hline Pneumonia bacterial & Infections and infestations & 636 & 11.5 & $10.5-12.5^{*}$ \\
\hline Pyrexia & General disorders and administration site conditions & 622 & 1.35 & $1.25-1.47^{*}$ \\
\hline Pulmonary tuberculosis & Infections and infestations & 573 & 27.9 & $25.3-30.9 *$ \\
\hline Cellulitis & Infections and infestations & 524 & 6.73 & $6.15-7.38 *$ \\
\hline Lymphoma & Neoplasms benign, malignant and unspecified & 454 & 14.9 & $13.5-16.5^{*}$ \\
\hline Pyelonephritis & Infections and infestations & 309 & 10.4 & $9.19-11.7 *$ \\
\hline Pancytopenia & Blood and lymphatic system disorders & 296 & 1.42 & $1.27-1.6 *$ \\
\hline Disseminated tuberculosis & Infections and infestations & 292 & 31.9 & $27.6-36.7^{*}$ \\
\hline Organising pneumonia & Infections and infestations & 261 & 13.6 & $11.9-15.5^{*}$ \\
\hline Hepatic function abnormal & Hepatobiliary disorders & 248 & 0.41 & $0.36-0.46$ \\
\hline Anaphylactoid reaction & Immune system disorders & 246 & 2.7 & $2.37-3.07^{*}$ \\
\hline Breast cancer & Neoplasms benign, malignant and unspecified & 241 & 12.5 & $10.9-14.3^{*}$ \\
\hline Urinary tract infection & Infections and infestations & 226 & 3.61 & $3.15-4.13 *$ \\
\hline White blood cell count decreased & Investigations & 219 & 0.26 & $0.23-0.3$ \\
\hline Tuberculosis & Infections and infestations & 218 & 32.7 & $27.7-38.6 *$ \\
\hline Tuberculous pleurisy & Infections and infestations & 216 & 47.4 & $39.6-56.7^{*}$ \\
\hline Pleurisy & Respiratory, thoracic and mediastinal disorders & 214 & 10.8 & $9.34-12.5^{*}$ \\
\hline Platelet count decreased & Investigations & 211 & 0.23 & $0.2-0.27$ \\
\hline Arthritis bacterial & Infections and infestations & 205 & 13.7 & $11.8-16^{*}$ \\
\hline Bronchitis & Infections and infestations & 199 & 4.65 & $4.02-5.37 *$ \\
\hline Intestinal obstruction & Gastrointestinal disorders & 182 & 4.71 & $4.05-5.48 *$ \\
\hline Blood pressure decreased & Investigations & 173 & 1.05 & $0.9-1.22$ \\
\hline Rash & Skin and subcutaneous tissue disorders & 172 & 0.68 & $0.59-0.79$ \\
\hline Gastric cancer & Neoplasms benign, malignant and unspecified & 164 & 6.07 & $5.17-7.13 *$ \\
\hline
\end{tabular}

Note: *Signal detected.

Abbreviations: $\mathrm{Cl}$, confidence interval; PT, preferred terms; ROR, reporting odds ratio; SOC, system organ class; TNF, tumor necrosis factor.

Table 4 The Top 10 Adverse Drug Events Associated with Infliximab

\begin{tabular}{|l|l|l|l|}
\hline \multirow{2}{*}{ PT } & \multicolumn{3}{|l|}{ Infliximab } \\
\cline { 2 - 4 } & $\mathbf{n}$ & ROR & $\mathbf{9 5 \%} \mathbf{~ C l}$ \\
\hline Pneumonia & 1254 & 5.04 & $4.75-5.34^{*}$ \\
Pneumocystis jirovecii pneumonia & 888 & 14.5 & $13.5-15.5^{*}$ \\
Interstitial lung disease & 799 & 1.78 & $1.66-1.92^{*}$ \\
Infusion related reaction & 679 & 58.6 & $53.4-64.3^{*}$ \\
Herpes zoster & 412 & 6.82 & $6.17-7.55^{*}$ \\
Sepsis & 409 & 3.79 & $3.43-4.19^{*}$ \\
Pulmonary tuberculosis & 384 & 32.2 & $28.7-36.1^{*}$ \\
Pyrexia & 381 & 1.62 & $1.47-1.8^{*}$ \\
Pneumonia bacterial & 323 & 10.5 & $9.37-11.8^{*}$ \\
Lymphoma & 306 & 18.4 & $16.3-20.8^{*}$ \\
\hline
\end{tabular}

Note: *Signal detected.

Abbreviations: $\mathrm{Cl}$, confidence interval; PT, preferred terms; ROR, reporting odds ratio. have additional biological effects against transmembrane $\mathrm{TNF}^{16-20}$ and Fc receptor-expressing cells. ${ }^{21,22}$ Several studies demonstrated the binding of infliximab, adalimumab, etanercept, or certolizumab to cell lines expressing transfected transmembrane TNF, and showed that the degree of cell binding was 3 -fold greater with infliximab or adalimumab than with etanercept or certolizumab. ${ }^{19,23}$ In addition, CD64 modulates the efficacy of infliximab both in vitro and ex vivo, whereas the presence of this receptor has no impact on the inhibitory activity of certolizumab-pegol, which lacks the Fc fragment. ${ }^{21,22}$ In order to reduce adverse effects by preventing interaction with the Fc receptors, ${ }^{24}$ certolizumab pegol was produced without the $\mathrm{Fc}$ region, removing the mechanism 
Table 5 The Top 10 Adverse Drug Events Associated with Etanercept

\begin{tabular}{|l|l|l|l|}
\hline \multirow{2}{*}{ PT } & \multicolumn{3}{|l|}{ Etanercept } \\
\cline { 2 - 4 } & n & ROR & 95\% CI \\
\hline Pneumonia & 625 & 5.61 & $5.16-6.09 *$ \\
Interstitial lung disease & 529 & 2.72 & $2.49-2.97 *$ \\
Pneumocystis jirovecii pneumonia & 209 & 6.88 & $5.98-7.9 *$ \\
Herpes zoster & 153 & 5.5 & $4.68-6.47^{*}$ \\
Sepsis & 117 & 2.37 & $1.98-2.85^{*}$ \\
Pyrexia & 111 & 1.05 & $0.87-1.27$ \\
Cellulitis & 106 & 5.55 & $4.58-6.74 *$ \\
Pneumonia bacterial & 104 & 7.17 & $5.89-8.72 *$ \\
Breast cancer & 87 & 17.8 & $14.3-22.1 *$ \\
Urinary tract infection & 83 & 5.7 & $4.58-7.09 *$ \\
\hline
\end{tabular}

Note: *Signal detected.

Abbreviations: $\mathrm{Cl}$, confidence interval; PT, preferred terms; ROR, reporting odds ratio.

Table 6 The Top 10 Adverse Drug Events Associated with Adalimumab

\begin{tabular}{|l|l|l|l|}
\hline \multirow{2}{*}{ PT } & \multicolumn{3}{|l|}{ Adalimumab } \\
\cline { 2 - 4 } & $\mathbf{n}$ & ROR & 95\% CI \\
\hline Pneumonia & 272 & 3.39 & $3-3.83^{*}$ \\
Interstitial lung disease & 238 & 1.72 & $1.51-1.96^{*}$ \\
Pneumocystis jirovecii pneumonia & 160 & 7.62 & $6.5-8.93^{*}$ \\
Herpes zoster & 115 & 5.99 & $4.97-7.21^{*}$ \\
Pyrexia & 114 & 1.58 & $1.31-1.9^{*}$ \\
Pulmonary tuberculosis & 104 & 23.9 & $19.5-29.1^{*}$ \\
Cellulitis & 92 & 7.01 & $5.69-8.63^{*}$ \\
Pneumonia bacterial & 86 & 8.59 & $6.93-10.7^{*}$ \\
Pancytopenia & 82 & 2.52 & $2.02-3.14^{*}$ \\
Sepsis & 79 & 2.32 & $1.86-2.9 *$ \\
\hline
\end{tabular}

Note: *Signal detected.

Abbreviations: $\mathrm{Cl}$, confidence interval; PT, preferred terms; ROR, reporting odds ratio.

dependent on the presence of that region. ${ }^{24}$ In addition, certolizumab pegol was a pegylated anti-TNF $\mathrm{F}(\mathrm{ab}$ ')2 fragment, ${ }^{25}$ with a structure different from that of other TNF-alfa inhibitors. However, it is possible that other factors contribute to infections. Further studies will be needed to clarify this.

Different from other TNF-alfa inhibitors, infliximab is a mouse/human chimeric monoclonal TNF-alfa inhibitor of the IgG1 isotype, ${ }^{25}$ so it requires intravenous drip infusion. In our results, infusion reaction was reported only for infliximab. In addition, anaphylactic reaction and reduction in blood pressure were noted with infliximab. These results are consistent with those of previous reports. ${ }^{26-28}$
Table 7 The Top 10 Adverse Drug Events Associated with Golimumab

\begin{tabular}{|l|l|l|l|}
\hline \multirow{2}{*}{ PT } & \multicolumn{3}{|l|}{ Golimumab } \\
\cline { 2 - 4 } & $\mathbf{n}$ & ROR & $\mathbf{9 5 \% ~ C I}$ \\
\hline Pneumonia & 366 & 7.39 & $6.63-8.24^{*}$ \\
Interstitial lung disease & 194 & 2.16 & $1.87-2.49^{*}$ \\
Pneumonia bacterial & 123 & 19.3 & $16-23$. * $^{*}$ \\
Pneumocystis jirovecii pneumonia & 100 & 7.18 & $5.88-8.77^{*}$ \\
Cellulitis & 78 & 9.06 & $7.22-11.4^{*}$ \\
Pyelonephritis & 70 & 20.6 & $16.2-26.2^{*}$ \\
Hepatic function abnormal & 70 & 1.14 & $0.9-1.45$ \\
Platelet count decreased & 57 & 0.62 & $0.48-0.8 \mathrm{I}$ \\
Liver disorder & 56 & 1.53 & $1.18-2^{*}$ \\
Sepsis & 55 & 2.45 & $1.88-3.2 I^{*}$ \\
\hline
\end{tabular}

Note: *Signal detected.

Abbreviations: $\mathrm{Cl}$, confidence interval; PT, preferred terms; ROR, reporting odds ratio.

Table 8 The Top 10 Adverse Drug Events Associated with Certolizumab Pegol

\begin{tabular}{|l|l|l|l|}
\hline \multirow{2}{*}{ PT } & \multicolumn{3}{|l|}{ Certolizumab Pegol } \\
\cline { 2 - 4 } & $\mathbf{n}$ & ROR & $\mathbf{9 5 \% ~ C I}$ \\
\hline Pneumonia & 208 & 10.9 & $9.44-12.7^{*}$ \\
Herpes zoster & 119 & 25.1 & $20.8-30.4^{*}$ \\
Interstitial lung disease & 84 & 2.33 & $1.87-2.9^{*}$ \\
Pneumocystis jirovecii pneumonia & 44 & 7.83 & $5.79-10.6^{*}$ \\
Cellulitis & 32 & 9.16 & $6.45-13^{*}$ \\
Pyelonephritis & 25 & 17.9 & $12-26.6^{*}$ \\
Pneumonia bacterial & 24 & 8.94 & $5.96-13.4^{*}$ \\
Sepsis & 21 & 2.32 & $1.51-3.58^{*}$ \\
Pyrexia & 20 & 1.04 & $0.67-1.61$ \\
Colon cancer & 19 & 17.5 & $11.1-27.6^{*}$ \\
\hline
\end{tabular}

Note: *Signal detected.

Abbreviations: $\mathrm{Cl}$, confidence interval; PT, preferred terms; ROR, reporting odds ratio.

As for malignancy, our results showed that lymphoma, breast cancer, and gastric cancer were significantly correlated with TNF-alpha inhibitors as a class. Especially, reports of breast cancer were numerous with etanercept. It has been reported that the immunosuppressive effects of TNF-alpha inhibitors raise the risk of cancer, ${ }^{29}$ although it is known that RA disease activity by itself is a risk factor for developing malignancy. ${ }^{30}$

Several studies reported that TNF-alfa inhibitors are associated with central nervous system (CNS) demyelinating disorders, as well as peripheral nervous system disorders (Guillain-Barré syndrome, Miller Fisher syndrome, chronic inflammatory demyelinating polyneuropathy, multifocal motor neuropathy with conduction 
block, mononeuropathy multiplex, and axonal sensorimotor polyneuropathies). ${ }^{31,32}$ However, in this study, the top 30 adverse events caused by TNF-alfa inhibitors included no CNS-related adverse events.

The present study has several limitations. First, this study does not cover all known TNF-alpha inhibitors. Second, as a consequence of the self-reporting database, it is difficult to completely remove reporting bias. In addition, the data occasionally contain coding errors. Third, ROR does not provide a robust indication of the signal strength. In spontaneous reporting systems such as JADER, control populations are not included, so ROR is different from the "odds ratio" that is commonly used in epidemiological studies. In real terms, ROR indicates an increased risk of adverse event reporting, and not the risk of an adverse event itself. Finally, the JADER database did not include detailed clinical information on the patients' clinical status.

In conclusion, the strength of the association among TNF-alfa inhibitors with adverse events is variable, and further studies are required to evaluate the identified signals.

\section{Author Contributions}

All authors contributed to data analysis, drafting or revising the article, gave final approval of the version to be published, and agreed to be accountable for all aspects of the work.

\section{Funding}

K. Hosohata received research support from the Science Research Promotion Fund.

\section{Disclosure}

The authors report no conflicts of interest in this work.

\section{References}

1. Ettehadi P, Greaves MW, Wallach D, Aderka D, Camp RD. Elevated tumour necrosis factor-alpha (tnf-alpha) biological activity in psoriatic skin lesions. Clin Exp Immunol. 1994;96:146-151. doi:10.1111/j.13652249.1994.tb06244.x

2. Sandborn WJ, Hanauer SB, Rutgeerts P, et al. Adalimumab for maintenance treatment of crohn's disease: results of the classic ii trial. Gut. 2007;56:1232-1239. doi:10.1136/gut.2006.106781

3. van de Kerkhof PC, Griffiths CE, Reich K, et al. Secukinumab long-term safety experience: A pooled analysis of 10 Phase II and iii clinical studies in patients with moderate to severe plaque psoriasis. J Am Acad Dermatol. 2016;75(83-98):e84. doi:10.1016/j.jaad.2016. 03.024

4. Smolen JS, Kay J, Doyle MK, et al. Golimumab in patients with active rheumatoid arthritis after treatment with tumour necrosis factor alpha inhibitors (go-after study): A multicentre, randomised, double-blind, placebo-controlled, phase iii trial. Lancet. 2009;374:210-221. doi:10.1016/S0140-6736(09)60506-7
5. Mahe J, de Campaigno EP, Chene AL, Montastruc JL, Despas F, Jolliet P. Pleural adverse drugs reactions and protein kinase inhibitors: identification of suspicious targets by disproportionality analysis from vigibase. Br J Clin Pharmacol. 2018. doi:10.1111/ bcp. 13693

6. Mendes D, Alves C, Batel-Marques F. Safety profiles of adalimumab, etanercept and infliximab: A pharmacovigilance study using a measure of disproportionality in a database of spontaneously reported adverse events. J Clin Pharm Ther. 2014;39:307-313. doi: $10.1111 /$ jept. 12148

7. Hosoya R, Uesawa Y, Ishii-Nozawa R, Kagaya H. Analysis of factors associated with hiccups based on the japanese adverse drug event report database. PLoS One. 2017;12:e0172057. doi:10.1371/journal. pone. 0172057

8. Kose E. Adverse drug event profile associated with pregabalin among patients with and without cancer: analysis of a spontaneous reporting database. J Clin Pharm Ther. 2018;43:543-549. doi:10.1111/jcpt.12683

9. Hosohata K, Inada A, Oyama S, Furushima D, Yamada H, Iwanaga K. Surveillance of drugs that most frequently induce acute kidney injury: A pharmacovigilance approach. J Clin Pharm Ther. 2019;44:49-53. doi:10.1111/jcpt.12748

10. Hosohata K, Inada A, Oyama S, Niinomi I, Wakabayashi T, Iwanaga $\mathrm{K}$. Adverse cutaneous drug reactions associated with oldand new- generation antiepileptic drugs using the japanese pharmacovigilance database. Clin Drug Investig. 2019;39:363-368. doi:10.1007/s40261-019-00754-z

11. Oyama S, Hosohata K, Inada A, et al. Drug-induced tubulointerstitial nephritis in a retrospective study using spontaneous reporting system database. Ther Clin Risk Manag. 2018;14:1599-1604. doi:10.2147/ TCRM.S168696

12. Inada A, Hosohata $\mathrm{K}$, Oyama $\mathrm{S}$, et al. Evaluation of medication-related osteonecrosis of the jaw using the japanese adverse drug event report database. Ther Clin Risk Manag. 2019;15:59-64. doi:10.2147/TCRM.S176620

13. Lane MA, McDonald JR, Zeringue AL, et al. Tnf-alpha antagonist use and risk of hospitalization for infection in a national cohort of veterans with rheumatoid arthritis. Medicine. 2011;90:139-145. doi:10.1097/MD.0b013e318211106a

14. Minozzi S, Bonovas S, Lytras T, et al. Risk of infections using anti-tnf agents in rheumatoid arthritis, psoriatic arthritis, and ankylosing spondylitis: A systematic review and meta-analysis. Expert Opin Drug Saf. 2016;15:11-34. doi:10.1080/14740338.2016.12 40783

15. Wakao R, Taavola H, Sandberg L, et al. Data-driven identification of adverse event reporting patterns for japan in vigibase, the who global database of individual case safety reports. Drug Safety. 2019;42:1487-1498. doi:10.1007/s40264-019-00861-y

16. Derer S, Till A, Haesler R, et al. Mtnf reverse signalling induced by tnfalpha antagonists involves a gdf-1 dependent pathway: implications for crohn's disease. Gut. 2013;62:376-386. doi:10.1136/gutjnl-2011300384

17. Kaymakcalan Z, Sakorafas P, Bose S, et al. Comparisons of affinities, avidities, and complement activation of adalimumab, infliximab, and etanercept in binding to soluble and membrane tumor necrosis factor. Clin immunol. 2009;131:308-316. doi:10.10 16/j.clim.2009.01.002

18. Nesbitt A, Fossati G, Bergin M, et al. Mechanism of action of certolizumab pegol (cdp870): in vitro comparison with other anti-tumor necrosis factor alpha agents. Inflamm Bowel Dis. 2007;13:1323-1332. doi:10.1002/ibd.20225

19. Scallon B, Cai A, Solowski N, et al. Binding and functional comparisons of two types of tumor necrosis factor antagonists. J Pharmacol Exp Ther. 2002;301:418-426. doi:10.1124/jpet.301.2.418

20. Shealy DJ, Cai A, Staquet K, et al. Characterization of golimumab, a human monoclonal antibody specific for human tumor necrosis factor alpha. mAbs. 2010;2:428-439. doi:10.4161/mabs.12304 
21. Vos AC, Wildenberg ME, Duijvestein M, Verhaar AP, van den Brink GR, Hommes DW. Anti-tumor necrosis factor-alpha antibodies induce regulatory macrophages in an fc region-dependent manner. Gastroenterology. 2011;140:221-230. doi:10.1053/j.gastro.2010.10.008

22. Wojtal KA, Rogler G, Scharl M, et al. Fc gamma receptor cd64 modulates the inhibitory activity of infliximab. PLoS One. 2012;7: e43361. doi:10.1371/journal.pone.0043361

23. Mitoma $\mathrm{H}$, Horiuchi $\mathrm{T}$, Tsukamoto $\mathrm{H}$. Binding activities of infliximab and etanercept to transmembrane tumor necrosis factor-alpha. Gastroenterology. 2004;126:934-935; author reply 935-936. doi:10. 1053/j.gastro.2004.01.036

24. Levin AD, Wildenberg ME, van den Brink GR. Mechanism of action of anti-tnf therapy in inflammatory bowel disease. J Crohn's Colitis. 2016;10:989-997. doi:10.1093/ecco-jcc/jjw053

25. Tracey D, Klareskog L, Sasso EH, Salfeld JG, Tak PP. Tumor necrosis factor antagonist mechanisms of action: A comprehensive review. Pharmacol Ther. 2008;117:244-279. doi:10.1016/j.pharmthera.2007. 10.001

26. Iwanczak BM, Ryzko J, Jankowski P, et al. Evaluation of the infliximab therapy of severe form of pediatric crohn's disease in poland: retrospective, multicenter studies. Adv Clin Exp Med. 2017;26:51-56. doi: $10.17219 /$ acem $/ 35802$
27. Filho AG, Kinote A, Pereira DJ, et al. Infliximab prevents increased systolic blood pressure and upregulates the akt/enos pathway in the aorta of spontaneously hypertensive rats. Eur $J$ Pharmacol. 2013;700:201-209. doi:10.1016/j.ejphar.2012.11.059

28. Choquette D, Faraawi R, Chow A, Rodrigues J, Bensen WJ, Nantel F. Incidence and management of infusion reactions to infliximab in a prospective real-world community registry. $J$ Rheumatol. 2015;42:1105-1111. doi:10.3899/jrheum.140538

29. Setoguchi S, Solomon DH, Weinblatt ME, et al. Tumor necrosis factor alpha antagonist use and cancer in patients with rheumatoid arthritis. Arthritis Rheum. 2006;54:2757-2764. doi:10.1002/art.22056

30. Simon TA, Thompson A, Gandhi KK, Hochberg MC, Suissa S. Incidence of malignancy in adult patients with rheumatoid arthritis: A meta-analysis. Arthritis Res Ther. 2015;17:212. doi:10.1186/ s13075-015-0728-9

31. Bosch X, Saiz A, Ramos-Casals M, Group BS. Monoclonal antibody therapy-associated neurological disorders. Nat Rev Neurol. 2011; 7:165-172. doi:10.1038/nrneurol.2011.1

32. Mohan N, Edwards ET, Cupps TR, et al. Demyelination occurring during anti-tumor necrosis factor alpha therapy for inflammatory arthritides. Arthritis Rheum. 2001;44:2862-2869. doi:10.1002/15290131(200112)44:12<2862::AID-ART474>3.0.CO;2-W
Therapeutics and Clinical Risk Management

\section{Publish your work in this journal}

Therapeutics and Clinical Risk Management is an international, peerreviewed journal of clinical therapeutics and risk management, focusing on concise rapid reporting of clinical studies in all therapeutic areas, outcomes, safety, and programs for the effective, safe, and sustained use of medicines. This journal is indexed on PubMed Central, CAS,

\section{Dovepress}

EMBase, Scopus and the Elsevier Bibliographic databases. The manuscript management system is completely online and includes a very quick and fair peer-review system, which is all easy to use. Visit http://www.dovepress.com/testimonials.php to read real quotes from published authors. 